\title{
Variabilidad genética de dos poblaciones de arroz permite la selección de plantas promisorias para la cosecha mecanizada
}

\section{Genetic variability of two rice populations allows the selection of promising plants for mechanized harvesting}

\section{Eldo Yoel Flores del Castillo', Gladys Julia Álvarez Paz ${ }^{2}$}

${ }^{1}$ MsC. Ingeniería Agrícola, Estación Territorial de Investigaciones de Granos Sur del Jíbaro (ETIG), ORCID: https://orcid.org/0000-00034399-2276, eldoyoel@gmail.com

2 Lic. Informática, Joven Club de Computación y Electrónica (JCCE) La Sierpe, ORCID: https://orcid.org/0000-0002-2428-9342, gladys. alvarez@ssp.jovenclub.cu

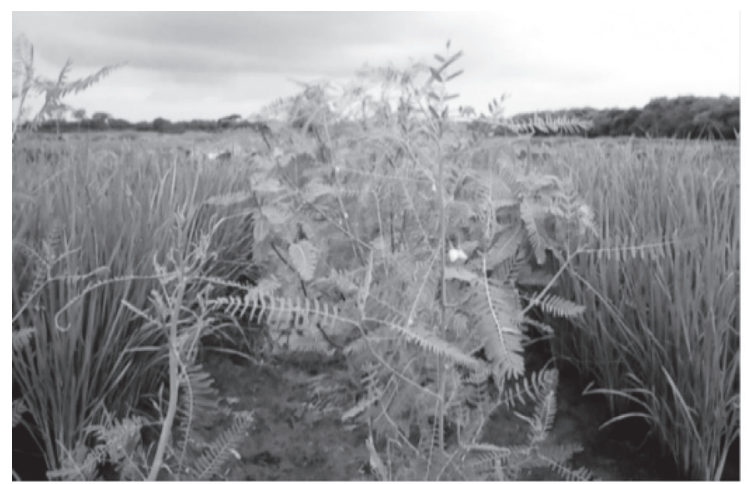

\section{RESUMEN}

En la estación de investigaciones de granos Sur del Jíbaro, en la provincia Sancti Spíritus, durante la campaña húmeda 2016-2017, se evaluaron dos poblaciones de arroz para determinar la variabilidad genética en base a sus caracteres morfo-agronómicos. PIACuba-4 y PIACuba-5 son poblaciones portadoras del gen de androesterilidad, obtenidas por mejora poblacional a través de la selección recurrente. Fueron sembradas en parcelas mediante la tecnología de trasplante, en un área de $700 \mathrm{~m}^{2}$. Para evaluar las plantas de forma individual se adoptó un marco de siembra de 0.30 x 0.25 metros, los caracteres evaluados fueron: longitud del tallo, rendimiento por planta, cantidad de granos por panícula, longitud de las panículas, número de hijos por planta, longitud de los granos y peso de mil granos. Los datos se procesaron mediante una estadística descriptiva para datos agrupados por distribución de frecuencias; en ambas poblaciones se observó variabilidad genética y esta varió en dependencia de la población y el carácter evaluado. Se logró determinar la variabilidad genética de las poblaciones PIACuba-4 y PIACuba-5, los caracteres que mostraron mayor coeficiente de variación fueron la longitud del tallo, el rendimiento por planta, el número de granos por panícula y el número de hijos por planta. Otros caracteres como la longitud de la panícula, longitud de los granos y peso de 1000 granos mostraron coeficientes de variación moderados. A partir de la evaluación morfo-agronómica de las poblaciones, se seleccionaron plantas promisorias con características apropiadas para la cosecha mecanizada y se colectaron semillas de 120 líneas seleccionadas, 66 originadas de la población PIACuba-4 y 54 derivadas de PIACuba-5. Palabras clave: androesterilidad, características, gen ms, líneas.

\begin{abstract}
At the Sur del Jíbaro grain research station, in Sancti Spíritus province, during the 2016-2017 wet season, two rice populations were evaluated to determine genetic variability based on their morphoagronomic characters. PIACuba- 4 and PIACuba-5 are populations carrying the androsterility gene, obtained by population improvement through recurrent selection. These, were planted in plots through transplant technology, in an area that included $700 \mathrm{~m}^{2}$. To evaluate the plants individually a seeding frame of $0.30 \times 0.25 \mathrm{~m}$ was adopted, the evaluated characters were: stem length, yield per plant, number of grains per panicle, length of the panicles, number of stems per plant, length of the grains and weight of 1000 grains. The data was processed by descriptive statistics for data grouped by distribution of frequency; in both populations, genetic variability was observed and this varied depending on the population and the evaluated character. The genetic variability of PIACuba- 4 and PIACuba-5 populations was determined, the characters that showed the highest coefficient of variation were stem length, yield per plant, number of grains per panicle and number of stem per plant. Other characters such as panicle length, grain length and weight of 1000 grains showed moderate coefficients of variation. From the morphoagronomic evaluation of the populations, promising plants with appropriate characteristics for the mechanized harvest were selected and seeds of 120 selected lines were collected, 66 originated from the PIACuba-4 population and 54 derived from PIACuba-5.
\end{abstract}

Keywords: Male sterility, characteristics, gene ms, lines.
Recibido: 7 de julio del 2019

Aceptado: 6 de septiembre del 2019

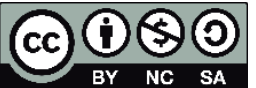

C Copyright 2019. Universidad Nacional Agraria
Los artículos de la revista La Calera de la Universidad Nacional Agraria, Nicaragua, se comparten bajo términos de la licencia Creative Commons: Reconocimiento, No Comercial, Compartir Igual. Las autorizaciones adicionales a las aquí delimitadas se pueden obtener en el correo edgardo.jimenez@ci.una.edu.ni 


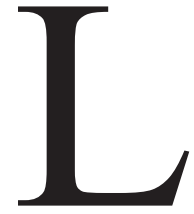

a importancia del arroz (Oryza sativa L.) como alimento para la sociedad cubana se puede comprender a partir del elevado consumo per cápita anual estimado en alrededor de los $60 \mathrm{~kg}$, muy por encima de casi todos los países del continente americano y cercano a los patrones de consumo de algunos países asiáticos (MINAG, 2014).

En Cuba, la estrategia varietal para el cultivo de arroz fue establecida en el año 1984 y posteriormente ha sido revisada en los años 2000 y 2012, donde se recomendaron variedades las cuales citamos a continuación: para la época de seca de ciclo corto con menos de 130 días (IACuba 25, IACuba 31, Perla, Reforma), ciclo medio entre 130 y 150 días (IACuba 32, IACuba 30, INCA-LP7, Selección 1, Selección 2), ciclo largo con más de 150 días Prosequisa 4 (MINAG, 2014).

La recolección es de interés general dentro del proceso tecnológico arrocero debido a que es el escalón intermedio entre la agricultura y la industria, influenciada por el impulso que la mecanización ha desarrollado en las últimas décadas. Resultados obtenidos de evaluaciones de la recolección mecanizada de arroz en Cuba, revelaron que la cosecha presenta de cinco a $6.5 \%$ de granos pelados y/o quebrados, y entre cinco y $10 \%$ de impurezas, valores estos por encima de los límites establecidos, por lo que afectan el rendimiento agrícola (NRAG-920: 88 y NRAG-910: 88, 2008). El exceso de granos dañados e impurezas en la cosecha, es causado entre otros factores por el cultivar empleado. Con el propósito a largo plazo de obtener cultivares de arroz que contribuyen a disminuir las pérdidas en la cosecha mecanizada, en la estación experimental de granos de la provincia de Sancti Spíritus se desarrolla un programa de mejoramiento genético de esta especie basado en la selección recurrente. Como fuente de androesterilidad genética para el desarrollo de poblaciones de base de arroz de riego, se utilizó un mutante del cultivar IR-36 portador del gen ms (Singh y Ikehashi, 1981). El objetivo general de este trabajo fue determinar la variabilidad genética de dos poblaciones de arroz portadoras del gen ms para seleccionar plantas promisorias con características apropiadas para la cosecha mecanizada.

\section{MATERIALES Y MÉTODOS}

Del segundo semestre de 1996 al primero del 2000 se introdujeron y evaluaron el acervo genético GPIRAT-10 y las poblaciones PCT-4 y PCT-7 procedentes del Centro Internacional de Agricultura Tropical de Colombia (CIAT) y a partir de ellas se desarrollaron la población PIACuba-4 para la cual se utilizó la población introducida PCT-7 como progenitor femenino, portadora del gen recesivo ms y cul- tivares cubanos IACuba-25, IACuba-31, IACuba-34, Perla de Cuba, INCA-LP-5 y FEDEARROZ-50, mientras que la PCT-7 cruzada con los cultivares cubanos IACuba-31, IACuba-34, IACuba-37, INCA-LP-5 y FEDEARROZ-50 dieron origen a la población PCT-5.

Estos cultivares que se emplearon como progenitores están bien adaptados a las condiciones edafoclimáticas de Cuba, además con características de importancia para los ámbitos de selección. Las semillas obtenidas fueron mezcladas y así quedaron formadas las poblaciones con siete y seis progenitores, además de poseer en sus bases genéticas genes foráneos procedentes de la población utilizada como progenitor femenino. Durante la campaña húmeda de 20162017, fueron sembradas las poblaciones PIACuba-4 y PIACuba-5 en la Estación de Investigaciones de Granos Sur del Jíbaro, provincia Sancti Spíritus ubicada en la latitud media de Cuba, a los $21^{\circ} 4^{\prime} 3^{\prime \prime}$ de latitud Norte y $79^{\circ} 11^{\prime} 6^{\prime \prime}$ de longitud Oeste con suelos Gley Vérticos típicos. La siembra de las poblaciones se realizó en semilleros, distanciados en el tiempo en término de siete días, manteniendo tal diferencia a la fecha de trasplante para permitir el cruzamiento de individuos con diferencias en el ciclo de floración, cada uno de estos fue trasplantado a los $25 \mathrm{ddg}$ (días de germinado) en un área que comprendía $700 \mathrm{~m}^{2}$, con una distancia de $0.30 \times 0.25$ $\mathrm{m}$, posibilitando evaluar plantas tomadas de un área elegida al azar; además las poblaciones se aislaron con una barrera de plantas de Sesbania rostrata Brem. y Oberm habilitando así un espacio entre poblaciones para evitar el cruzamiento natural entre estas y con plantas foráneas. Se cosecharon en cada una de las poblaciones 200 plantas, manteniéndose así las características de éstas para posteriores siembras según lo recomendaron Geraldi y Souza, (2000). Utilizando el sistema de evaluaciones estándar para arroz del IRRI (2013), fueron evaluados siete caracteres sobre la base de una muestra de 100 plantas de cada población en varios surcos de un área al azar; los caracteres evaluados fueron:

- Longitud del tallo: (LT) en cm; se obtuvo midiendo desde la base de la planta hasta el ápice de la panícula del tallo más alto.

- Rendimiento por planta: $(\mathrm{R} / \mathrm{P})$ en g; consistió en tomar muestras de plantas en cada población y realizar un pesaje del total de granos llenos producidos por cada una de ellas, para el pesaje se utilizó una balanza digital Metter Toledo con precisión de $0.10 \mathrm{mg}$.

- Número de granos por panícula: (NG); se realizó con un contador de granos láser (SLY-C).

- Longitud de las panículas: (LP) en cm; se midió la longitud de las panículas, desde la inserción de ésta hasta el ápice del último grano, para lo cual se utilizó una regla graduada. 
- Número de hijos por planta: (NH); se refiere al número de hijos menos uno producido por la planta.

- Longitud de los granos: (LG) en mm; por la parte longitudinal del grano se midió la longitud de granos normales tomados al azar con la ayuda de un pie de rey digital marca Mitutoyo.

- Peso de 1000 granos: (P1000G) en g; se realizó el pesaje de 1000 granos ajustados al 14\% de humedad en muestras de cada población, este se separó en siete clases diferentes desde 20 a 35 g. Para el pesaje se utilizó una balanza digital Metter Toledo, con precisión de $0.10 \mathrm{mg}$ y un determinador de humedad PM-450.

Las labores culturales durante el ciclo de las poblaciones tales como preparación del terreno, siembra, fertilización y riego se efectuaron, según lo establecido en el Instructivo Técnico del Cultivo del Arroz (MINAG, 2014). La información disponible fue procesada mediante el paquete estadístico STATGRAPHICS Plus v 5.1 sobre Windows.

\section{RESULTADOS Y DISCUSIÓN}

Longitud del tallo. La frecuencia de aparición de la longitud del tallo, varió desde 43 hasta $110 \mathrm{~cm}$ en las muestras colectadas de las poblaciones PIACuba-4 y PIACuba-5. En las dos poblaciones se observó predominio de la longitud del tallo en el rango de 61 a $90 \mathrm{~cm}$ (Figura 1). Cuando se comparó la longitud del tallo, en el rango de 71 a 80 $\mathrm{cm}$ de las plantas originadas de cada población, se observó que la frecuencia relativa de aparición fue superior en PIACuba-4. En las dos poblaciones objeto de estudio se presentaron pocas plantas con longitud del tallo entre 101 y $110 \mathrm{~cm}$. En la totalidad de la muestra colectada a partir de PIACuba-4 y PIACuba-5, la frecuencia de plantas con longitud de tallo inferior a $60 \mathrm{~cm}$ estuvo por debajo del $15 \%$ y se clasificaron como semienana acorde al sistema de evaluación estándar propuesto por (IRRI, 2013). La longitud del tallo es uno de los caracteres de gran importancia para la cosecha mecanizada y el rendimiento del cultivo, pues está directamente relacionada con el acame de las plantas (Ospina, Guimarães, Chatel y Duque, 2003). En el presente experimento las dos poblaciones estudiadas presentaron un rango de variación extenso.
Rendimiento por planta. El promedio nacional de rendimiento en el cultivo de arroz en Cuba, no ha sobrepasado las $3.6 \mathrm{t} \mathrm{ha}^{-1}$ pese al potencial productivo de los cultivares obtenidos por el programa nacional de mejoramiento genético (PNMG) y de la existencia de tecnologías capaces de garantizar resultados de producción satisfactorios. El número de productores que rebasan las cuatro y cinco $t$ ha $^{-1}$ cada vez aumentan más, lo que demuestra que existen posibilidades de elevar la productividad de este cultivo en el país (MINAG, 2014). Los componentes de rendimiento pueden verse limitados debido a la influencia climática, densidad de siembra y aplicación de fertilizantes entre otros factores (Sheehy, Dionora y Mitchell, 2001). En esta investigación, para las dos poblaciones estudiadas, las plantas con rendimiento en el rango de 20 a 40 g presentaron las mayores frecuencias.

En PIACuba-5 se observó mayor frecuencia de plantas que produjeron de 20-30 gramos de granos, mientras que en PIACuba-4, el mayor porcentaje de las plantas muestreadas rindieron de 30 a 40 gramos (Figura 2).

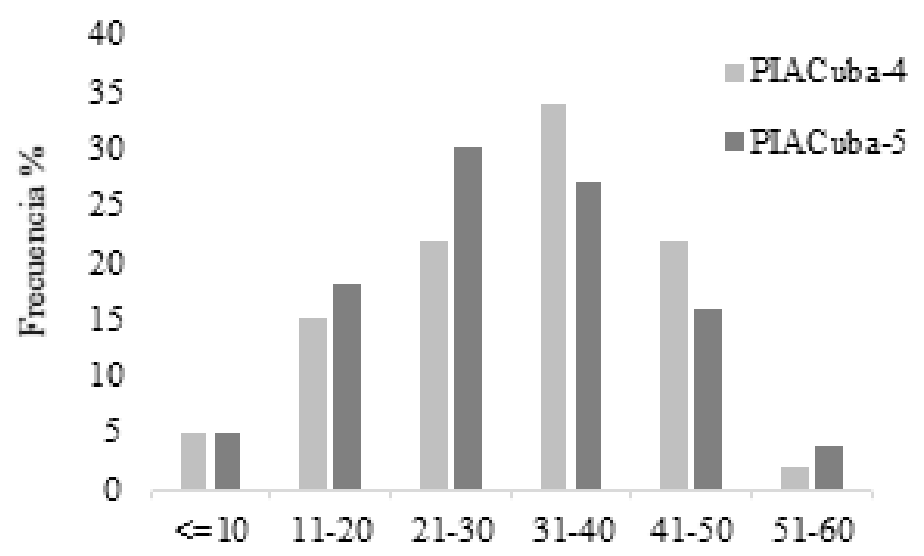

Figura 2. Distribución de frecuencias para el rendimiento $(\mathrm{g})$ por plantas. 
Similar para ambas poblaciones entre el $15 \mathrm{y}$ $17 \%$ de las plantas muestreadas generaron de 10 a $20 \mathrm{~g}$ de arroz y el 5\% produjeron menos de $10 \mathrm{~g}$. Sin embargo, en la población PIACuba-5 la frecuencia de plantas que produjeron hasta $60 \mathrm{~g}$ de arroz fue superior a la observada en PIACuba-4, lo que significa que para una siembra por trasplante como la realizada en el experimento resulta un rendimiento de $5.3 \mathrm{tha}^{-1}$.

Según Vidal, Bezus, Pinciroli y Scelzo (2015), existen países con programas de mejoramiento genético en arroz, que poseen líneas promisorias con características diferenciadas, las cuales radican en menor altura y mayor potencial de ahijamiento, además con estructura de planta más compacta, lo que les brinda un mejor comportamiento agronómico para obtener altos rendimientos.

Número de granos por panícula. Se observó que existe alta variabilidad en el número de granos por panícula. Se detectaron plantas con panículas desde menos de 60 hasta 260 granos. En las dos poblaciones del total de muestra colectada, las plantas que produjeron entre 101 y 140 granos por panícula se presentaron en mayor frecuencia. Tanto para las plantas de PIACuba-4 como PIACuba-5, el número de granos por panícula en los rangos de 60-100 y 141-181 presentaron frecuencias entre 17 y $22 \%$. En la población PIACuba-4 la frecuencia de plantas con 181-220 granos por panícula fue superior que en PIACuba-5. Sin embargo, el porcentaje de plantas con 221-250 granos por panícula fue similar en las dos poblaciones (Figura 3). El número de granos por panícula constituye uno de los factores fundamentales en el rendimiento del arroz. Pero, un número elevado de granos por panículas cuando la planta no posee tallos fuertes puede provocar acercamiento de la panícula al suelo y aumentar la humedad del grano.

Cuando las panículas se acercan al suelo, se produce mayor cantidad de fisuras en los granos en el momento de la cosecha por daño mecánico. Es fundamental que los genotipos con alto número de granos por panícula posean tallos gruesos y fuertes para que soporten el peso de las panículas.

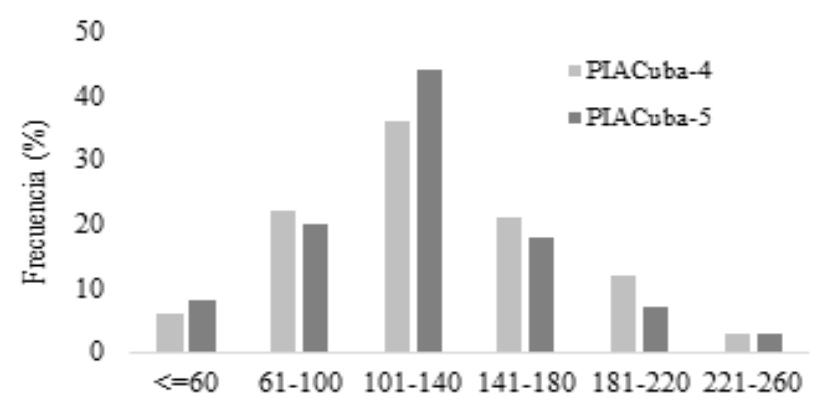

Figura 3. Distribución de frecuencias para el número de granos por panículas.

Soto (1991), informó que el número de granos por panícula estuvo determinado principalmente por dos aspectos, el número de ramificaciones y el largo que ésta haya alcanzado. En el arroz, el número de granos por panícula oscila entre 50 y 500 granos, aunque en la mayoría de las variedades comerciales oscila entre 100 y 150 granos por panícula. Para producir genotipos que nos ofrezcan un rendimiento alto es necesario que estos presenten un número considerable de granos por panícula.

Longitud de las panículas. Las poblaciones PIACuba-4 y PIACuba-5 presentaron plantas que producen panículas desde 19 hasta $32 \mathrm{~cm}$. En las dos poblaciones la longitud de la panícula en el rango de 24 a $26 \mathrm{~cm}$ se presentó con mayor frecuencia, seguida por las longitudes de 26-28 y 23-24 cm. En la población PIACuba-5 el porcentaje de plantas que emitieron panículas con longitudes entre $30 \mathrm{y}$ $32 \mathrm{~cm}$ fue dos veces superior a las observadas en PIACuba-4. De acuerdo al descriptor IRRI (2013), la longitud de la panícula para las poblaciones PIACuba-4 y PIACuba-5 se clasifican como media y larga (Figura 4).
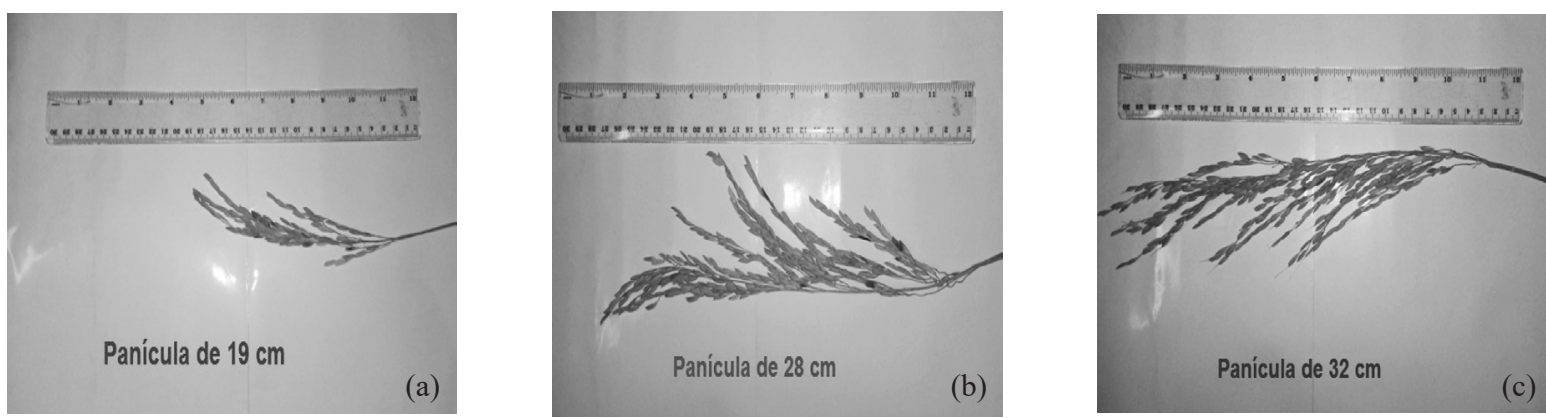

Figura 4. Panículas en las poblaciones PIACuba-4 y PIACuba-5. (a) panícula media (b) panícula larga (c) panícula larga. 
Las variaciones en la longitud de la panícula están ligadas a los progenitores y son altamente influenciadas por el ambiente (Sarawgi, Subba, Parikh, Sharma y Ojha, 2013). Esta influencia de los progenitores sobre longitud de la panícula pudo ser la causa de la estabilidad de este carácter.

Número de hijos por planta. El número de hijos por planta presentó un intervalo que abarcó desde plantas con menos de tres hijos hasta plantas con 15 hijos. Del total de la muestra colectada tanto para PIACuba-4 como para PIACuba-5 las plantas con siete a nueve hijos presentaron mayor frecuencia (Figura 5).

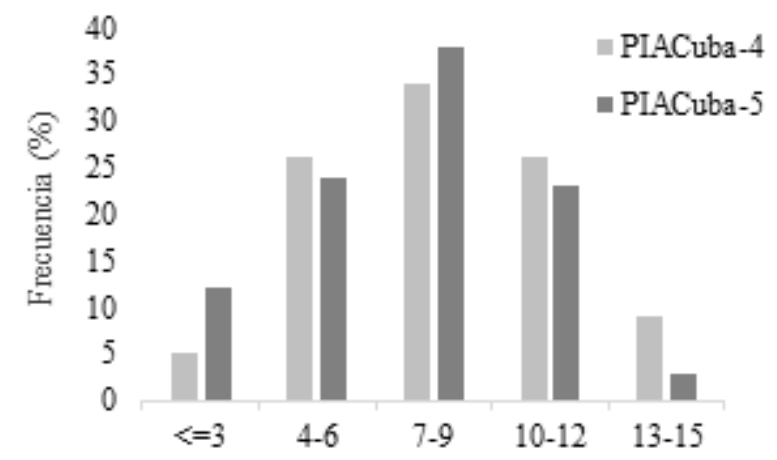

Figura 5. Distribución de frecuencias para el número de hijos por plantas.

De forma similar en ambas poblaciones, la frecuencia descendió para las clases donde las plantas emitieron de cuatro a seis y de 10 a 12 hijos. Es de destacar que la frecuencia de plantas con bajo ahijamiento $(\leq 3)$ fue dos veces mayor en la población PIACuba-5 que en PIACuba-4. Sin embargo, la frecuencia de plantas con alto ahijamiento fue tres veces superior en la población PIACuba-4 que en PIACuba-5. Aunque aproximadamente el $85 \%$ de las plantas muestreadas presentaron de cuatro a 12 hijos, se observó un amplio rango para esta variable que mostró alta desviación típica. Ello es muy favorable, pues permite seleccionar plantas para diferentes agrotécnias ya sea siembra directa o trasplante.

La obtención de plantas con tallos fuertes para sistemas de riego por aniego, que resistan el peso de largas panículas y que se opongan al acamado, son importante para disminuir las pérdidas en la cosecha. Olmos (2007), informó que el número de hijos depende de la densidad de plantación y varia en dependencia de esta; en alta densidad de siembra, las plantas de arroz emitieron tres hijos, mientras que en bajas densidades formaron 15 hijos. En línea con los resultados de este trabajo, las poblaciones estudiadas fueron originadas de varios progenitores y ello debió generar la respuesta variada en el ahijamiento.

Longitud de los granos. La longitud del grano varió desde $\leq 5 \mathrm{~mm}$ hasta $12 \mathrm{~mm}$ (Figura 6).

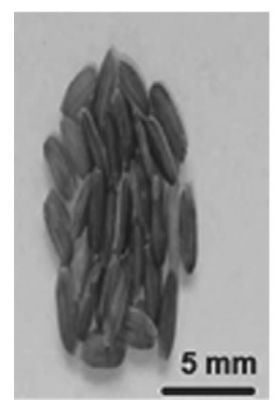

(a)

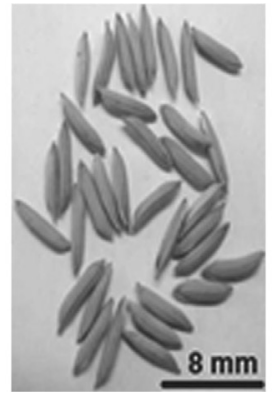

(b)

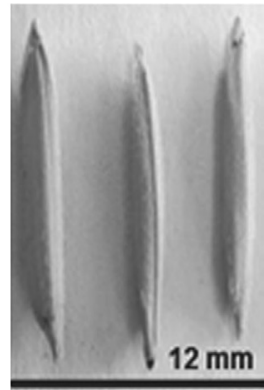

(c)
Figura 6. Longitudes de granos de PIACuba-4 y PIACuba-5; (a) Granos cortos, (b) granos intermedios, (c) granos extra largos.

En la población PIACuba-5 se observó mayor de granos con longitudes entre ocho y ocho punto nueve $\mathrm{mm}$ que en PIACuba-4. Sin embargo, las plantas que rindieron granos en el rango de 10-10.9 mm fueron más frecuentes en la población PIACuba-4 que en PIACuba-5. Para ambas poblaciones se observaron frecuencias similares de plantas con granos extra largos, mientras que presencia de plantas con granos cortos en PIACuba-4 fue dos veces mayor que en PIACuba-5. El carácter longitud del grano no presentó amplia variabilidad, pero el material genético obtenido mostró valores que son preferidos por los mejoradores en Cuba, granos de ocho milímetros. Por lo general los granos largo y extra largo tienden a partirse menos durante la cosecha que un grano corto grueso con alto contenido de almidón (Degiovanni, Cesar, Martínez y Motta, 2010).

De ahí que la longitud del grano tiene alta influencia en la calidad industrial del arroz y por ello es un aspecto a tener en cuenta por los fitomejoradores. Aunque no se observó alta variabilidad en este carácter, si se obtuvieron plantas con rangos de longitud variables: cortos, intermedios y extralargos.

Peso de 1000 granos. Para cultivares cubanos el peso de mil granos fluctúa entre 25 y 30 gramos; en las poblaciones estudiadas este carácter varió desde 20 hasta 33 gramos y el rango más frecuente fue de 24 a $29 \mathrm{~g}$. La frecuencia de peso de 1000 granos para los rangos de clase de 22-23 g y 30-31 g disminuyó considerablemente para las dos poblaciones; así como las clases extremas tanto inferior como superior (Figura 7). 


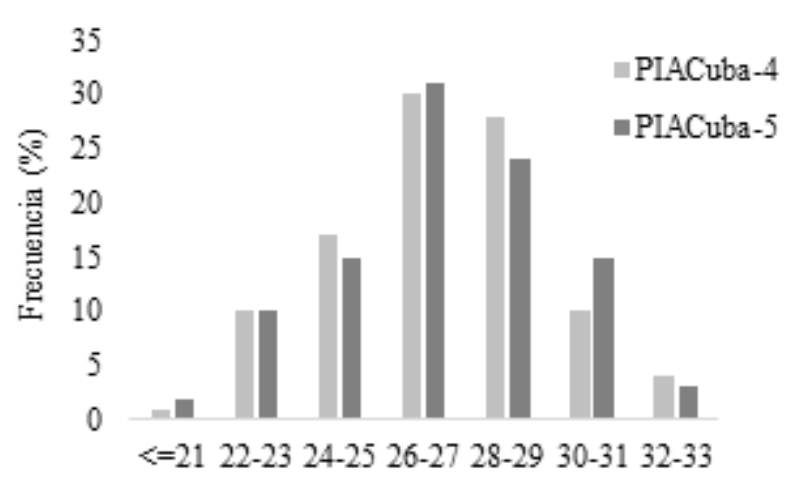

Figura 7. Distribución de frecuencia para el peso de 1000 granos (g) en ambas poblaciones.

El comportamiento del carácter peso de 1000 granos fue muy similar al carácter longitud del grano, ambos están muy relacionados, pues es evidente que la alta frecuencia observa en un rango de peso de 26 a 29 gramos está en correspondencia con la frecuencia de granos largos (nueve a $9.9 \mathrm{~mm}$ de longitud). Por consiguiente, el peso de 1000 granos superior a los 30 gramos fue originado por granos de longitud extra larga, mientras que el peso de 1000 granos inferior a los 24 gramos está en correspondencia con la producción de granos cortos. Las dos poblaciones mostraron amplia variación para dicho carácter. En este caso no estuvo influenciado por rangos de temperatura distintos, la causa de su variabilidad debió ser genética, pues el peso de 1000 granos es una característica muy estable y con alta heredabilidad. En el arroz uno de los objetivos fundamentales es obtener cultivares que expresen un alto potencial de rendimiento con el cual a la vez se podrá elevar la productividad de la maquinaria.

Para la diferenciación de la variabilidad de los caracteres evaluados, se aplicó una estadística descriptiva para datos agrupados por distribuciones de frecuencias a través de la cual se pudieron detectar los caracteres que mayor y menor variabilidad genética aportaron a las poblaciones. Tanto para PIACuba-4 como PIACuba-5 los caracteres longitud del tallo, rendimiento por planta, número de granos por panícula y número de hijos por planta presentaron alto coeficiente de variación por lo que mostraron amplia variabilidad genética. En el caso de la longitud de los granos y el peso de 1000 granos, el coeficiente de variación fue moderado y su variabilidad genética inter- media. El carácter longitud de la panícula resultó con un coeficiente de variación bajo, por lo que mostró poca variabilidad genética (Cuadro 1).

Cuadro1. Coeficiente de variación expresado en (\%) para cada carácter evaluado

\begin{tabular}{cccccccc}
\hline Poblaciones & LT & R/P & NG & NH & LP & LG & P1000G \\
\hline PIAC-4 & 22.3 & 39.8 & 36.7 & 41.3 & 8.2 & 10.6 & 10.0 \\
\hline PIAC-5 & 20.6 & 37.1 & 46.0 & 37.5 & 8.3 & 10.8 & 10.2 \\
\hline
\end{tabular}

*LT: Longitud del tallo, R/P: Rendimiento por planta, NG: Número de granos por panículas, LP: Longitud de las panículas, NH: Número de hijos por planta, LG: Longitud de los granos, P1000G: Peso de mil granos.

El coeficiente de variación genotípico $(\mathrm{CVG})$ y el coeficiente de variación fenotípico (CVF) son útiles para detectar la variabilidad presente en el germoplasma (Idris, Justin, Dagash y Abual, 2012). Los coeficientes CVG se clasifican como bajos cuando se encuentran en el rango de cero a $10 \%$, moderados de 10 a $20 \%$ y altos $>20 \%$. En general, un alto coeficiente de variación indica que hay una amplia variabilidad genética que conduce a un buen ámbito de selección e incrementa las posibilidades de mejora; por otro lado, valores bajos de coeficiente de variación revelan la necesidad de incrementar la variabilidad genética.

\section{CONCLUSIONES}

Se consiguió determinar la variabilidad genética de las poblaciones PIACuba-4 y PIACuba-5, los caracteres que mostraron mayor coeficiente de variación fueron la longitud del tallo, el rendimiento por planta, el número de granos por panícula y el número de hijos por planta, además, se logró la selección de plantas promisorias con características apropiadas para la cosecha mecanizada y se colectaron semillas de 120 líneas seleccionadas, 66 originadas de la población PIACuba-4 y 54 derivadas de PIACuba-5. 


\section{REFERENCIAS BIBLIOGRÁFICAS}

Degiovanni, V., Cesar, P., Martínez, R., y Motta, O. (2010). Producción eco-eficiente del arroz en América Latina. Tomo I. Capítulos 1-24. ISBN 978-958-694-103-7. Disponible en http://ciat-library.ciat.cgiar.org/Articulos_Ciat/2010_Degiovanni-Produccion_ecoeficiente_del_arroz.pdf

Geraldi, O.P., y Souza, J.R. (2000). Muestreo genético para programas de mejoramiento. En: Guimarães, E P (ed). Avances en el mejoramiento poblacional en arroz. Centro Internacional de Agricultura Tropical (CIAT), Cali. Colombia, pp. 9-19.

Idris, A. E., Justin, F. J., Dagash, M. I., y Abual, A. I. (2012). Genetic Variability and Inter Relationship between Yield and Yield Components in Some Rice Genotypes. American Journal of Experimental Agriculture, 2(2),233-239. Disponible en: http://sustech. edu/staff_publications/2012040914492313.pdf

IRRI (Standard Evaluation System for Rice, 5th Edition). Sistema de evaluación estándar para el arroz. Los Baños, Philippines. (2013). 46 p. Disponible en http://www.knowledgebank.irri.org/images/docs/rice-standard -evaluation-system.pdf

MINAG (Ministerio de la agricultura). (2014). Instructivo Técnico del cultivo del arroz. ISBN: 978-959-7210-86-3. La Habana, Cuba, $40 \mathrm{p}$.

NRAG-920: 88 y NRAG-910: 88. (2008). Arroz cáscara húmedo. Determinación de materias extrañas, La Habana, Cuba.

Olmos, S. (2007). Apunte de morfología, fenología, eco fisiología, y mejoramiento genético del arroz. Cátedra de Cultivos II, Facultad de Ciencias Agrarias, UNNE, Corrientes - Argentina. Disponible en http://www.acpaarrozcorrientes.org.ar/academico/ApunteMORFOLOGIA.pdf

Ospina, Y., Guimarães, E. P., Chatel, M., y Duque, C. (2003). Efectos de la selección y recombinación en una población de arroz de secano. En E. Guimarães (ed). Mejoramiento poblacional, una alternativa para explorar los recursos genéticos del arroz en América Latina. Cap. 17: 355-374.

Sarawgi, A., Subba, K. V., Parikh, M., Sharma, B., y Ojha, G. C. (2013). Assessment of Variability of Rice (Oryza sativa L.). Germoplasm using Agro-morphological Characterization. Journal of Rice Research, 6(1), 14-20. Disponible en http://www.icariirr.org/assesment.pdf

Sheehy, J., Dionora., y Mitchell, M. (2001). Spikelet numbers, sink size and potential yield in rice. Field crops research, (71), pp. 77-85. Disponible en: https://www.researchgate.net/publication/222150689_Spikelet_numbers_sink_size_and_potential_yield_in_rice

Singh, R.J., y Ikehashi, H.I. (1981). Monogenic male sterility in rice: Induction, identification and inheritance. Crop.Science, 2 (21), pp 286-289. Disponible en https://dl.sciencesocieties.org/publications/cs/abstracts/21/2/CS0210020286

Soto, B. (1991). Estudio de observación de 20 variedades USA y siete líneas Promisorias nacionales en comparación con dos testigos comerciales de arroz, Managua, Nicaragua.

Vidal, A., Bezus, R., Pinciroli, M., y Scelzo, L. (2015). Rendimiento y Calidad de Líneas de Arroz Largo/Fino, especiales y Largo/ Ancho del Programa Arroz UNLP, campaña 2014/15. Jornadas Proarroz INTA. Concordia. 